\title{
Fase da lesão cerebral e o diagnóstico cinético- funcional de sujeitos com paralisia cerebral
}

\author{
Phase of brain injury and the functional kinetic diagnosis of individuals with \\ cerebral palsy
}

\footnotetext{
1 (iD) Universidade Federal do Rio Grande do Sul (UFRGS), Porto Alegre, RS, Brasil.

daiaherther@hotmail.com

2 (D) Programa de Pós-Graduação Saúde da Criança e do Adolescente, Universidade Federal do Rio Grande do Sul (UFRGS), Porto Alegre, RS, Brasil.

gerzson.lais@yahoo.com.br

3 (iD) Departamento de Fisioterapia, Educação Física e Dança, Universidade Federal do Rio Grande do Sul (UFRGS), Porto Alegre, RS, Brasil.

carlaskilhan@gmail.com
}

\author{
Daiana Seffrin Herther ${ }^{1}$ \\ Laís Rodrigues Gerzson ${ }^{2}$ \\ Carla Skilhan de Almeida ${ }^{3}$ \\ Endereço para correspondência \\ Carla Skilhan de Almeida \\ Rua Felizardo, 750 - Jardim Botânico \\ 90690-200 - Porto Alegre - RS Brasil \\ carlaskilhan@gmail.com
}

\begin{abstract}
Resumo
Introdução: A paralisia cerebral (PC) é caracterizada por uma lesão no SNC ainda em fase de desenvolvimento, não progressiva, levando a limitações funcionais. As causas da PC são multifatoriais e ocorrem nas fases pré, peri ou pós-natal, até o segundo ano de vida da criança.

Objetivo: Analisar a correlação entre a fase de lesão cerebral e o diagnóstico cinéticofuncional de crianças com PC.

Método: Estudo retrospectivo, com revisão de 131 prontuários de pacientes de um ano e um mês aos 18 anos atendidos em um Centro de Reabilitação.

Resultados: Fase de lesão de maior prevalência foi a perinatal $(43,5 \%)$; espasticidade bilateral foi o diagnóstico cinético-funcional mais prevalente $(68,7 \%)$; foi encontrada correlação significativa entre a fase da lesão e diagnóstico motor $(p=0,013)$. Conclusão: Fase de lesão pré-natal teve maior correlação em sujeitos com espasticidade bilateral; fase de lesão perinatal correlacionou-se com os diagnósticos de espasticidade unilateral, mista e atáxica e, fase de lesão pós-natal teve maior correlação com a discinética.
\end{abstract}

Descritores: Paralisia Cerebral. Lesões Encefálicas. Tono Muscular.

\begin{abstract}
Introduction: Cerebral palsy (CP) is characterized by a non-progressive developmental lesion in the CNS, leading to functional limitations. The causes of CP are multifactorial and occur in the pre, peri or postnatal phases, up to the second year of the child's life.

Aim: To analyze the correlation between the phase of cerebral lesion and the functional kinetic diagnosis of children with CP.

Method: Retrospective study, with review of 131 medical records of patients from one year and one month to 18 years of age attended at a Rehabilitation Center. Results: The most prevalent lesion phase was perinatal $(43.5 \%)$; bilateral spasticity was the most prevalent kinetic-functional diagnosis (68.7\%); a significant correlation was found between the lesion stage and motor diagnosis $(\mathrm{p}=0.013)$.

Conclusion: Phase of prenatal lesion had a higher correlation in subjects with bilateral spasticity; perinatal lesion phase correlated with unilateral, mixed and ataxic spasticity, and the postnatal lesion phase had a higher correlation with dyskinetic.
\end{abstract}

Keywords: Cerebral Palsy. Brain Injuries. Muscle tonus.

Cite como

Herther DS,Gerzson LR, Almeida CS. Fase da lesão cerebral e o diagnóstico cinético-funcional de sujeitos com paralisia cerebral. Conscientiae Saúde 2019 jul./set.; 18(3):352-365. https://doi.org/10.5585/ConsSaude.v18n3 14176. 


\section{Introdução}

A paralisia cerebral (PC) é caracterizada por uma lesão no Sistema Nervoso Central (SNC) ainda em fase de desenvolvimento, de forma não progressiva, que causa disfunções cognitivas, de linguagem, sociais, motoras, entre outras. Como disfunção motora, podemos encontrar alterações de tônus, de movimento, de postura e distúrbios músculo esqueléticos, levando a limitações funcionais da criança. Essas disfunções podem vir acompanhadas de crises convulsivas, distúrbios sensoriais, de comunicação, cognição, comportamento, assim como complicações respiratórias e gastrointestinais. ${ }^{1,2,3}$

A incidência da PC varia de acordo com o desenvolvimento de cada país. Mas, a estimativa mundial reporta a prevalência de PC que varia entre 1.5 a 4 crianças por 1,000 nascidos vivos. ${ }^{4}$ Devido ao menor acesso aos serviços de saúde em países subdesenvolvidos, como no Brasil, aproximadamente sete crianças a cada 1.000 nascidas são acometidas. ${ }^{5} \mathrm{Um}$ estudo publicado pelo Centre Disease Control and Prevention (Centro de Controle de Doença e Prevenção) mostrou que a PC é mais comum em meninos e em crianças negras. As crianças hispânicas e brancas têm a mesma probabilidade de ter PC. A maioria $(77,4 \%)$ das crianças identificadas com PC apresentava PC espástica. Mais da metade (58,2\%) das crianças podem andar de forma independente. Muitas outras crianças podem apresentar mais de uma condição (41\% tinham epilepsia concomitante e 6,9\% tinham transtorno do aspecto autista - TEA). ${ }^{4,6}$

As lesões acometem o encéfalo (ainda imaturo) até dois anos de idade e as causas da PC são multifatoriais. Como causas pré-natais, destacam-se as infecções, parasitoses, traumatismos no abdômen, queda da gestante, malformações cerebrais do feto e causas não especificadas inicialmente, que levam o feto a entrar em sofrimento (Nonreassuring Fetal Status - NRFS) ${ }^{7}$. Destaca-se o NRFS, pois este é uma das maiores causas de PC e acontece na transição da fase fetal com a fase perinatal, ou seja, no final da gestação e início do trabalho de parto. Ocorre uma alteração tecidual em que o feto recebe menos alimento e oxigênio. Pode ser por causas de pouco líquido amniótico, cordão umbilical pinçado, problemas na mãe (cardíacos, pulmonares, pressão arterial alta ou baixa, descolamento de placenta, contração uterina muito forte e frequente), uso de alguma medicação ou posicionamento da mãe que diminua o fluxo sanguíneo para o feto. ${ }^{7}$

Como causas perinatal, a literatura destaca principalmente a prematuridade devido à hemorragia intraventricular (HIV) e as causas sentinelas ${ }^{8}$ como: ruptura de placenta, prolapso de cordão, ruptura uterina, embolismo do fluido amniótico, parto laborioso, causando uma isquemia ou sangramento no cérebro do bebê nas primeiras horas após o nascimento). ${ }^{9}$ Nas 
causas pós-natais, encontram-se os distúrbios metabólicos, meningite, hiperbilirrubinemia, traumatismos crânio encefálicos, desnutrição e o acidente vascular cerebral (AVC). A lesão cerebral varia, dependendo, principalmente, do tempo do lapso e da extensão da lesão. A avaliação inicial do cérebro após a lesão é desafiador, pois há uma incerteza para saber em que momento ocorreu o evento neurológico. Os exames complementares (eletroencefalograma, ecografía cerebral, líquido cefalorraquidiano, ressonância magnética) e a clínica do paciente são grandes aliados para o diagnóstico precoce..$^{10,11}$

A classificação da PC é feita de acordo com o local da lesão no SNC e da sintomatologia da topografia corporal. Segundo a classificação Européia de PC, baseada no Surveillance of Cerebral Palsy in Europe (SCPE) ela é dividida em: em espástica (lesão piramidal), discinética (lesão extrapiramidal), atáxica (lesão cerebelar) ou mista (lesão piramidal e extra-piramidal). As formas mistas são caracterizadas por diferentes combinações de transtornos motores pirâmido-extrapiramidais, pirâmido-atáxico ou pirâmido-extrapiramidal-atáxicos. ${ }^{12}$ Também pode ser encontrado na forma hipotônica, porém é mais rara. Quanto à topografia, a PC pode ser bilateral ou em unilateral. ${ }^{13,14} \mathrm{O}$ tipo bilateral é caracterizado por comprometimento dos quatro membros (conhecida como quadriplegia) ou somente dos membros inferiores (diplegia), devido à lesão encefálica bilateral, extensa, simétrica ou não. Já o tipo unilateral pode ser conhecido como uma hemiplegia ou monoplegia. ${ }^{15}$

O comprometimento motor dos sujeitos com PC vai depender da área lesada, da gravidade e da extensão em que ocorreu a lesão cerebral. Quanto maior for esta lesão, maiores serão as limitações e as restrições funcionais que esta criança vai apresentar, dificultando, assim a aquisição de habilidades. O nascimento prematuro, por exemplo, pode influenciar e interromper o neurodesenvolvimento típico, trazendo consequências negativas para o cérebro da criança. Acontecimentos perinatais, tipo de parto, apgar, histórico gestacional e possíveis traumas devem ser levados em consideração. ${ }^{16,17}$

O que se sabe com mais clareza é que a lesão pode ocorrer nos períodos pré-natais, perinatais ou pós-natais, como citado anteriormente. O que ainda se procura é se existe uma correlação da fase de lesão cerebral e o diagnóstico cinético-funcional de uma criança com PC. Talvez esta correlação possa predizer ou indicar o que mais vem interessando os investigadores atuais: como o SNC do feto e do bebê com lesão pode desenvolver-se. Mais ainda, como a lesão do SNC pode afetar a funcionalidade do sujeito no futuro. Para isso, muitas vezes, devemos analisar o passado para tentar predizer esse futuro. Por isso, baseados neste questionamento, o 
presente estudo teve como objetivo correlacionar a fase de lesão cerebral (pré, peri e pós-natal) com o diagnóstico cinético-funcional das crianças com Paralisia Cerebral em um Centro de Reabilitação.

\section{Métodos}

Delineamento

Trata-se de um estudo retrospectivo, realizado por meio da revisão de 131 prontuários, de pacientes do ambulatório de Reabilitação de um Centro de Integração de Criança Especial de Porto Alegre (KINDER), em janeiro de 2019.

\section{Amostra}

A amostra deste projeto teve como critérios de inclusão: todas as crianças e adolescentes até 18 anos de idade com diagnóstico de Paralisia Cerebral que frequentavam a KINDER. Foram excluídos do estudo sujeitos que não apresentaram PC como diagnóstico clínico, que ultrapassavam a idade limite de 18 anos ou aquelas que apresentavam síndromes genéticas como diagnóstico de base.

\section{Instrumento de coleta de dados}

Este estudo teve como instrumento para coleta de dados os prontuários das crianças com diagnóstico de PC que frequentavam a Instituição. As crianças são submetidas a uma avaliação global de triagem, realizada pela equipe interdisciplinar ao ingressarem na instituição. Para compor as duas variáveis (fase de lesão e diagnóstico cinético-funcional atual), os dados de histórico gestacional das crianças e a avaliação atual da equipe foram explorados.

\section{Procedimento de coleta de dados}

Primeiramente, foram selecionadas as crianças que eram compatíveis com os critérios de inclusão. Após, as informações colhidas dos prontuários foram armazenadas em banco de dados com os dados gerais dos pacientes, a avaliação institucional com diagnóstico clínico, diagnóstico cinético-funcional e a história pregressa. Por fim, os dados foram analisados. 
Forma de análise de dados

Foi utilizado o software Statistical Package for the Social Sciences (SPSS) versão 25.0 para fazer a tabulação dos dados que foram analisados de forma descritiva, através de frequências e prevalências. A fase da lesão cerebral foi correlacionada com o diagnóstico cinético-funcional através do Coeficiente de Correlação Qui-quadrado de Pearson. O nível de significância utilizado foi de $95 \%$.

Questões Éticas

O presente estudo foi submetido a um "Termo de autorização para pesquisa em prontuário"; disponibilizado pela Coordenação de Reabilitação da Instituição. Este estudo teve a aprovação do Comitê de Ética da Universidade Federal do Rio Grande do Sul sob parecer número: 3.120.622 (CAAE: 96765618.5.0000.5347).

\section{Resultados}

O número de crianças com o diagnóstico de paralisia cerebral foi de 131 no total, dos quais, 56 crianças classificadas como pré-natal $(42,7 \%), 57$ classificadas como fase perinatal (43,5\%, sendo mais de $60 \%$ destes em razão da prematuridade) e 18 na fase pós-natal $(13,7 \%)$. Também, as frequências mais encontradas dos diagnósticos cinéticos funcionais nas crianças estudadas (Tabela 1). 
Tabela 1 - Frequências e Porcentagens relacionadas à fase de lesão e dos diagnósticos cinéticos funcionais.

\begin{tabular}{|c|c|c|c|}
\hline & & $\begin{array}{l}\text { Frequência } \\
\text { (n) }\end{array}$ & $\begin{array}{l}\text { Porcentagem } \\
(\%)\end{array}$ \\
\hline \multirow[t]{3}{*}{ Fase da lesão } & Pré & 56 & 42,7 \\
\hline & Peri & 57 & 43,5 \\
\hline & Pós & 18 & 13,7 \\
\hline \multirow{8}{*}{$\begin{array}{l}\text { Diagnósticos } \\
\text { cinéticos } \\
\text { funcionais }\end{array}$} & Total & 131 & 100,0 \\
\hline & Unilateral & 7 & 5,3 \\
\hline & Bilateral & 90 & 68,7 \\
\hline & Mista & 8 & 6,1 \\
\hline & Ataxia & 13 & 9,9 \\
\hline & Discinética & 5 & 3,8 \\
\hline & Hipotonia & 8 & 6,1 \\
\hline & Total & 131 & 100,0 \\
\hline
\end{tabular}

A Tabela 2 faz a associação do diagnóstico cinético-funcional com o período de lesão. Foi encontrada associação significativa entre a fase da lesão e o diagnóstico motor $(p=0,013)$. A fase pré-natal associou-se com a espasticidade bilateral $(48,9 \%)$. A fase perinatal associouse com a espasticidade unilateral $(85,7 \%)$, mista $(50 \%)$ e atáxica $(53,8 \%)$. Com relação à fase pós-natal, a associação foi com sujeitos que possuem um diagnóstico de discinesia (60\%). A hipotonia não se associou com nenhuma fase, mas ocorreu de forma equilibrada nas três fases. 
Tabela 2 - Associação do diagnóstico cinético funcional com o período de lesão

\begin{tabular}{|c|c|c|c|c|c|c|}
\hline \multirow[t]{30}{*}{ Diagnóstico } & \multirow{5}{*}{$\begin{array}{l}\text { Espasticidade } \\
\text { unilateral }\end{array}$} & Contagem & $0_{a}$ & $6 \mathrm{~b}$ & $1_{\mathrm{a}, \mathrm{b}}$ & 7 \\
\hline & & Contagem Esperada & 3,0 & 3,0 & 1,0 & 7,0 \\
\hline & & \% em Diagnóstico & $0,0 \%$ & $85,7 \%$ & $14,3 \%$ & $100,0 \%$ \\
\hline & & \% em Fase lesão & $0,0 \%$ & $10,5 \%$ & $5,6 \%$ & $5,3 \%$ \\
\hline & & $\%$ do Total & $0,0 \%$ & $4,6 \%$ & $0,8 \%$ & $5,3 \%$ \\
\hline & \multirow[t]{5}{*}{ Espasticidade bilateral } & Contagem & $44_{a}$ & $37 \mathrm{a}$ & $9 \mathrm{a}$ & 90 \\
\hline & & Contagem Esperada & 38,5 & 39,2 & 12,4 & 90,0 \\
\hline & & \% em Diagnóstico & $48,9 \%$ & $41,1 \%$ & $10,0 \%$ & $100,0 \%$ \\
\hline & & \% em Fase lesão & $78,6 \%$ & $64,9 \%$ & $50,0 \%$ & $68,7 \%$ \\
\hline & & $\%$ do Total & $33,6 \%$ & $28,2 \%$ & $6,9 \%$ & $68,7 \%$ \\
\hline & \multirow[t]{5}{*}{ Mista } & Contagem & $3 a$ & $4 a$ & $1_{\mathrm{a}}$ & 8 \\
\hline & & Contagem Esperada & 3,4 & 3,5 & 1,1 & 8,0 \\
\hline & & \% em Diagnóstico & $37,5 \%$ & $50,0 \%$ & $12,5 \%$ & $100,0 \%$ \\
\hline & & \% em Fase lesão & $5,4 \%$ & $7,0 \%$ & $5,6 \%$ & $6,1 \%$ \\
\hline & & $\%$ do Total & $2,3 \%$ & $3,1 \%$ & $0,8 \%$ & $6,1 \%$ \\
\hline & \multirow[t]{5}{*}{ Ataxia } & Contagem & $5 \mathrm{a}$ & $7 \mathrm{a}$ & $1_{\mathrm{a}}$ & 13 \\
\hline & & Contagem Esperada & 5,6 & 5,7 & 1,8 & 13,0 \\
\hline & & \% em Diagnóstico & $38,5 \%$ & $53,8 \%$ & $7,7 \%$ & $100,0 \%$ \\
\hline & & \% em Fase lesão & $8,9 \%$ & $12,3 \%$ & $5,6 \%$ & $9,9 \%$ \\
\hline & & $\%$ do Total & $3,8 \%$ & $5,3 \%$ & $0,8 \%$ & $9,9 \%$ \\
\hline & \multirow[t]{5}{*}{ Discinética } & Contagem & $2 a, b$ & $\mathrm{Ob}_{\mathrm{b}}$ & $3 \mathrm{a}$ & 5 \\
\hline & & Contagem Esperada & 2,1 & 2,2 &, 7 & 5,0 \\
\hline & & \% em Diagnóstico & $40,0 \%$ & $0,0 \%$ & $60,0 \%$ & $100,0 \%$ \\
\hline & & \% em Fase lesão & $3,6 \%$ & $0,0 \%$ & $16,7 \%$ & $3,8 \%$ \\
\hline & & $\%$ do Total & $1,5 \%$ & $0,0 \%$ & $2,3 \%$ & $3,8 \%$ \\
\hline & \multirow[t]{5}{*}{ Hipotonia } & Contagem & $2 \mathrm{a}$ & $3 a$ & $3 \mathrm{a}$ & 8 \\
\hline & & Contagem Esperada & 3,4 & 3,5 & 1,1 & 8,0 \\
\hline & & \% em Diagnóstico & $25,0 \%$ & $37,5 \%$ & $37,5 \%$ & $100,0 \%$ \\
\hline & & \% em Fase lesão & $3,6 \%$ & $5,3 \%$ & $16,7 \%$ & $6,1 \%$ \\
\hline & & $\%$ do Total & $1,5 \%$ & $2,3 \%$ & $2,3 \%$ & $6,1 \%$ \\
\hline \multirow[t]{5}{*}{ Total } & & Contagem & 56 & 57 & 18 & 131 \\
\hline & & Contagem Esperada & 56,0 & 57,0 & 18,0 & 131,0 \\
\hline & & \% em Diagnóstico & $42,7 \%$ & $43,5 \%$ & $13,7 \%$ & $100,0 \%$ \\
\hline & & \% em Fase lesão & $100,0 \%$ & $100,0 \%$ & $100,0 \%$ & $100,0 \%$ \\
\hline & & $\%$ do Total & $42,7 \%$ & $43,5 \%$ & $13,7 \%$ & $100,0 \%$ \\
\hline
\end{tabular}

Esp: espástica 
A Figura 1 mostra a associação entre a fase da lesão e o diagnóstico motor.

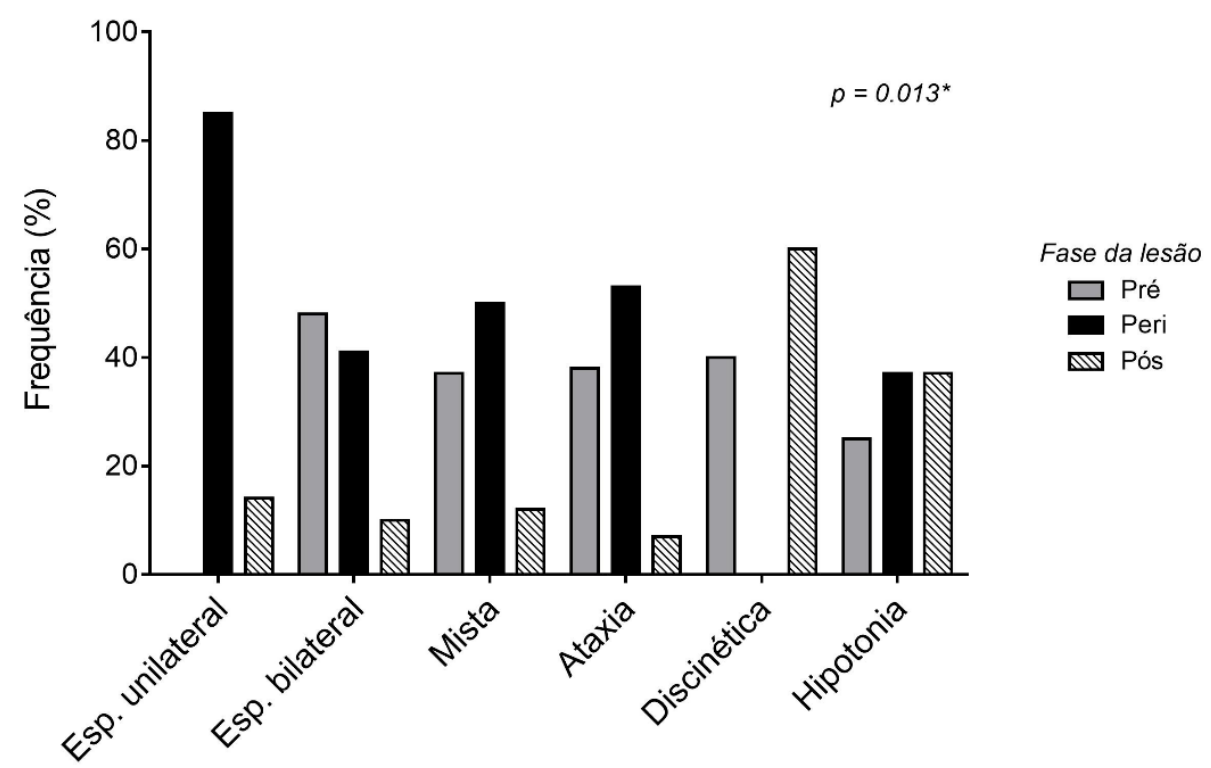

\section{Discussão}

A neuropatologia das lesões na PC difere de acordo com o grau de maturação cerebral, a época da lesão e a sua extensão. A maturação do SNC e o desenvolvimento de uma criança estão conectados com o tempo e é um processo longo. Esse processo depende de várias questões relacionadas com o desenvolvimento biológico, cognitivo, comportamental, emocional, social e principalmente como ele se integra com o ambiente. Predizer como será essa criança no seu futuro seria de grande pretensão que, talvez em estudos mais avançados, alcançaríamos. Porém, pode-se tentar correlacionar o momento da lesão do SNC de um bebê com características funcionais no futuro. Nosso estudo resolveu estudar essa correlação da fase de lesão do SNC no início da vida com o diagnóstico funcional da criança com PC, definido por uma equipe completa de reabilitação. Isso poderia aproximar uma relação de que determinada lesão no recém-nascido geraria determinada sequela.

Um estudo recente ${ }^{18}$ acompanhou 38 crianças por sete anos, onde associou os resultados da ressonância magnética na fase de lesão no início da vida (área cerebral acometida) com o diagnóstico cinético-funcional. Um dos resultados encontrados foi que a lesão cerebral grave pode ocorrer em um bebê recém-nascido após uma gravidez sem intercorrências. Este estudo mostrou também que as áreas acometidas nestes pacientes que desenvolveram PC foram: o córtex motor primário, as áreas semiovais e cápsula interna. Crianças com lesões que pouparam o sistema motor primário não desenvolveram PC. 
Corroborando com os dados encontrados na nossa coleta, estudos indicam que cerca de $30 \%$ dos casos de PC acontecem por causa pré-natal; $60 \%$ a $70 \%$ dos casos por causa perinatal devido à prematuridade, isquemias e sangramentos nas primeiras horas de vida e, eventos pósnatal está entre 10 e 21\%.8, ${ }^{8,9}$ A sequela de espasticidade é a mais frequente encontrada na literatura, acometendo mais de $70 \%$ dos casos, ${ }^{16,20}$ principalmente associada com a topografia bilateral, assim como em nosso estudo. A espasticidade ocorre devido à lesão hipóxicoisquêmica e sangramentos, principalmente da artéria cerebral média (ACM) nas áreas corticais e subcorticais, classicamente consideradas como sistema motor primário, como giros précentral, cápsula interna e substância branca. ${ }^{18,21}$

Encontramos no estudo a correlação entre a fase pré-natal e a espasticidade bilateral. Acredita-se que essas lesões extensas ocorram por eventos isquêmicos ou por sangramento bilateralmente no cérebro do feto. Esse cérebro está muito imaturo e mais suscetível a essas lesões. Lesões entre a $26^{\mathrm{a}}$ e $30^{\mathrm{a}}$ semana de gestação causam alterações principalmente na substancia periventricular e lesões que ocorre entre 32 e 36 semanas são lesões em córtex e núcleos da base. ${ }^{22}$ A isquemia é a mais grave forma de privação de $\mathrm{O}_{2}$. A encefalopatia hipóxicoisquêmica associada a alterações metabólicas, principalmente do metabolismo da glicose, levam a alterações bioquímicas, biofísicas e fisiológicas, que resultam no comprometimento do encéfalo. ${ }^{22}$ Quanto mais imaturo for o cérebro na sua fase de lesão, maior será o acometimento motor e funcional destes sujeitos.

Para a espasticidade unilateral, Santos sugere que a prevalência seja de $21-40 \%$ dos casos de PC e, 75\% delas, são de causas pré-natal, ${ }^{22}$ diferente dos resultados encontrados neste estudo, onde mostra a espasticidade unilateral em apenas 5,3\% das crianças ao total. A espasticidade unilateral teve uma correlação com a fase perinatal em 85,7\%, sem nenhuma correlação prénatal. Acredita-se que a espasticidade unilateral ocorra na fase perinatal devido a uma lesão hipóxico-isquêmica ou sangramentos de origem vascular cerebral, com déficit neurológico focal ou global unilateral, prolongado, de início agudo, gerando uma hemiplegia, podendo ser visualizado em imagens de ressonância magnética, principalmente em prematuros. ${ }^{18,23}$ Quanto mais prematuro for, maior será a prevalência desse tipo de lesão. ${ }^{20}$

A imaturidade que afeta o SNC do prematuro tem uma escassa capacidade de adaptação pós-natal. Existe uma fragilidade da estrutura vascular em nível da matriz germinativa e escassa migração neuronal, pobre mielinização da substância branca e crescimento exponencial da substância cinzenta. A suscetibilidade à hipóxia, alterações na osmolaridade e tensão, torna o 
sangramento em nível subepidérmico frequente com a produção de HIV, forma mais grave de infarto hemorrágico. Sua frequência em prematuros com peso inferior a $750 \mathrm{~g}$ excede $50 \%$, enquanto diminui para $10 \%$ naqueles com peso superior a $1.250 \mathrm{~g} \cdot{ }^{24,25}$ No presente estudo, mais de $60 \%$ das crianças que tiveram a lesão em fase perinatal foram prematuras e desenvolveram paralisia cerebral, corroborando com estudos da Sociedade Espanhola de Pediatria que afirmam que a prematuridade é a maior causa de PC. ${ }^{26}$

Além de a espasticidade unilateral estar correlacionada com a fase perinatal, a forma mista e atáxica (21\%) também se correlacionaram com esta fase. É comum os bebês prematuros terem regiões com isquemias e sangramentos difusos em núcleos da base, tálamo, cerebelo e vias corticoespinhais, ou seja, sequelas como espasticidade e discinesias. ${ }^{17,18,20}$ Corroborando com estes achados, Dinomais et al., encontraram na fase perinatal 14 (38\% da amostra) crianças com PC unilateral, destes, quatro tinham danos em regiões como a cápsula interna $(n=7)$, no giros pós-central do córtex motor primário $(\mathrm{n}=5)$. Na forma mista, quatro $(10 \%)$ tinham lesões em gânglios de base e tálamo. ${ }^{18}$

Com relação à fase pós-natal, foi a discinesia que encontramos correlação (60\%). Um dos fatores pós-natal que desenvolveu a PC nos pacientes estudados no presente estudo foi a hiperbilirrubinemia. ${ }^{27}$ A Kernicterus é uma anormalidade caracterizada por uma deposição excessiva de bilirrubina na região parenquimatosa central do cérebro, incluindo núcleos da base, tálamo e hipocampo. A bilirrubina é tóxica para os neurônios. Existem várias situações que podem levar a esse quadro, tais como incompatibilidade de Rh e deficiências enzimáticas. ${ }^{22}$ Ao serem lesados, os núcleos da base desenvolvem um quadro motor de PC discinética.

Este e outros estudos mostram a importância do acompanhamento de bebês de risco em programas de seguimento, de forma precoce. Também a importância de registrar de forma completa os dados da criança desde o começo. Por exemplo, o maior limitador deste estudo foi a dificuldade de encontrar à fase em que ocorreu a lesão cerebral nas crianças com PC. O histórico gestacional e história do parto colhidos nos prontuários, poderiam se enquadrar tanto na fase pré-natal, perinatal como na pós-natal. Foi necessária uma interpretação dos prontuários, conversas com as famílias e com a equipe de reabilitação. Tampouco existiam registros de exames complementares. Outra dificuldade observada foi à falta de informações quanto ao diagnósticos cinético-funcionais com nomenclaturas atualizadas. Os diagnósticos médicos não eram precisos, e existe uma tendência de assumir uma causa pré-natal na ausência de evidências claras de uma causa perinatal e pós-natal. ${ }^{19}$ 
O presente estudo tem alguns pontos fortes notáveis. Primeiro, usamos um número amostral razoável, de uma instituição reconhecida como uma das melhores de nossa cidade. Além disso, verificou-se a importância do diagnóstico cinético-funcional das crianças associando com a fase da lesão, o que dá indícios a outros profissionais verificarem possíveis prognósticos de crianças com lesões semelhantes, o mais cedo possível. Discutiu-se áreas anatômicas com as prováveis lesões que nossos pacientes apresentaram. No entanto, a contribuição mais importante, está no fato de que, se o bebê mostra indícios de lesão pré, peri ou pós natal, já realizar precocemente uma ecografia cerebral fontanelar ${ }^{28}$ ou ressonância magnética e encaminhá-la para um centro de intervenção motora precoce. Sugere-se para os próximos estudos uma associação com o contexto que esta criança está inserida e o quão precoce iniciou a intervenção.

\section{Conclusão}

A fase de lesão de maior prevalência foi a perinatal, muito por conta dos prematuros; a espasticidade bilateral foi o diagnóstico cinético-funcional mais prevalente; a fase de lesão prénatal teve maior correlação em sujeitos com espasticidade bilateral; a fase de lesão perinatal correlacionou-se com os diagnósticos de espasticidade unilateral, mista e atáxica e, a fase de lesão pós-natal teve maior correlação com a discinética.

Salientamos a importância de seguir um padrão de avaliação por parte das equipes interdisciplinares nos métodos de classificação e atualização nas nomenclaturas usadas, a fim de não apresentar divergências nas informações registradas em prontuários. Também sugerimos sempre registrar um histórico completo inicial e a evolução da criança no decorrer dos anos em prontuários.

Sugerimos criar seguimentos aos bebês de risco, principalmente para os bebês prematuros, bebês com lesões congênitas e bebês com lesões cerebrais pós-natais, para que possamos acompanhar seu prognóstico e melhorar sua funcionalidade. 


\section{Referências}

1. Wotherspoon J, Whittingham K, Boyd RN, Sheffield J. Randomised controlled trial of a novel online cognitive rehabilitation programme for children with cerebral palsy: a study protocol. BMJ Open. 2019;9(6):e028505.

2. Kwon HY, Kim BJ.Correlation between the dimensions of diaphragm movement, respiratory functions and pressures in accordance with the gross motor function classification system levels in children with cerebral palsy. J Exerc Rehabil. 2018;14(6):998-1004.

3. Cooper MS, Mackay MT, Fahey M, Reddihough D, Reid SM, Williams K. Seizures in Children With Cerebral Palsy and White Matter Injury. Pediatrics. 2017;139(3). pii: e20162975.

4. CDC - Centre Disease Control and Prevention. Data and Statistics for Cerebral Palsy. Page last reviewed: March 9, 2018. Acesso em: 12/06/2019.

https://www.cdc.gov/ncbddd/cp/data.html

5. Brasil. Ministério da Saúde. Secretaria de Atenção à Saúde. Departamento de Ações Programáticas Estratégicas. Diretrizes de atenção à pessoa com paralisia cerebral / Ministério da Saúde, Secretaria de Atenção à Saúde, Departamento de Ações Programáticas Estratégicas. - Brasília: Ministério da Saúde, 2013.

6. Autism and Developmental Disabilities Monitoring Network Surveillance Year 2008 Principal Investigators; Centers for Disease Control and Prevention. Prevalence of autism spectrum disorders--Autism and Developmental Disabilities Monitoring Network, 14 sites, United States, 2008. MMWR Surveill Summ. 2012;61(3):1-19.

7. Committee on Obstetric Practice, American College of Obstetricians and Gynecologists. ACOG Committee Opinion. Number 326, December 2005. Inappropriate use of the terms fetal distress and birth asphyxia. Obstet Gynecol. 2005;106(6):1469-70.

8. Martinez-Biarge M, Madero R, González A, Quero J, García-Alix A. Perinatal morbidity and risk of hypoxic-ischemic encephalopathy associated with intrapartum sentinel events. Am J Obstet Gynecol. 2012;206(2):148.e1-7.

9. Stephan-Otto C, Núñez C, Arca G, Agut T, García-Alix A. Three-Dimensional Map of Neonatal Arterial Ischemic Stroke Distribution From Early Multimodal Brain Imaging. Stroke. 2017;48(2):482-485.

10. Filho PCN, Duarte FT, Fortes JPA, Santos FFU. Alterações no comportamento elétrico cerebral de uma criança com Paralisia Cerebral após atendimento com neurofeedback. Fisioterapia Brasil 2017;18(3):369-373. 
11. Echeverría-Palacio CM, Agut T, Arnaez J, Valls A, Reyne M, Garcia-Alix A. Neuron-Specific Enolase in Cerebrospinal Fluid Predicts Brain Injury After Sudden Unexpected Postnatal Collapse. Pediatr Neurol. 2019; pii: S0887-8994(18)31326-2.

12. Surveillance of Cerebral Palsy in Europe. Urveillance of cerebral palsy in Europe: a Collaboration of cerebral palsy surveys andregisters. Surveillance of Crebral Palsy in Europe (SCPE). Dev Med Child Neurol. 2000;42(12):816-24.

13. Oliveira LS, Golin MO. Técnica para redução do tônus e alongamento muscular passivo: efeitos na amplitude de movimento de crianças com paralisia cerebral espástica ABCS Health Sci. 2017; 42(1):27-33.

14. Grave FMB, Aguiar LR, Ribeiro NMS. O kinesio taping no tratamento da paralisia cerebral. Fisioter Bras 2016;17(2):158-163.

15. Rosenbaum P, Paneth N, Leviton A, Goldstein M, Bax M, Damiano D et al. A report: the definition and classification of cerebral palsy, April 2006. Med Child Neurol. 2007;49(6):480

16. Agut T, Póo P, Launes C, Auffant M, Iriondo M. Incidence of cerebral palsy in a cohort of preterm infants with a gestational age of less than 28 weeks. An Pediatr (Barc).

2015;82(1):49-50.

17. García P, San Feliciano L, Benito F, García R, Guzmán J, Salas S,et al. Evolución a los 2 años de edad corregida de una cohortede recién nacidos con peso inferior o igual a $1.500 \mathrm{~g}$ de los gru-pos pertenecientes a la red neonatal SEN1500. An Pediatr (Barc). 2013;79(5):279-87.

18. Dinomais M, Hertz-Pannier L, Groeschel S, Chabrier S, Delion M, Husson B, et al; Long term motor function after neonatal stroke: Lesion localization above all. Hum Brain Mapp. 2015;36(12):4793-807.

19. Lino AM. Intervenção da fisioterapia na paralisia cerebral: relatório de estágio Lisboa. [Dissertação]. Escola Superior de Tecnologia da Saúde de Lisboa/Instituto Politécnico de Lisboa; 2016.

20. Ávila ASC, Rocha CAQ. Atuação fisioterapêutica em paciente com PC com tetraparesia espástica assimétrica: um estudo de caso. Rev Científica Da Faminas. 2014;10(2):21-27.

21. Ministerio de Sanidad, Servicios Sociales e Igualdad. Guía de Práctica Clínica sobre Encefalopatía Hipóxico-Isquémica Perinatal en el Recién Nacido, España, 2015. Disponível em: www.guiasalud.es/GPC/GPC_535_EHI_AQUAS_compl.pdf. Acesso em 16/06/2019.

22. Santos AF. Paralisia Cerebral: uma revisão da literatura. Unimontes Cientifica. 2015;16(2): 67-82.

23. Balachandran A, Kalyanshettar S, Patil S, Shegji V. Ischemic Stroke in Confederation with Trivial Head Trauma. Case Rep Pediatr. 2016; 2016: 2572958. 
24. Arca G, Arnaez J, Agut T, Núñez C, Stephan-Otto C, Valls A, et al. Neuron-specific enolase is correlated with lesion topology, relative infarct volume and outcome of symptomatic NAIS. Arch Dis Child Fetal Neonatal Ed. 2019;pii: fetalneonatal-2018-316680.

25. Olivé G, Agut T, Echeverría-Palacio CM, Arca G, García-Alix A. Usefulness of Cranial Ultrasound for Detecting Neonatal Middle Cerebral Artery Stroke. Ultrasound Med Biol. 2019;45(3):885-890.

26. Organización Mundial de La Salud. Nacimientos Prematuros. 2018. Disponível em : https://www.who.int/es/news-room/fact-sheets/detail/preterm-birth. Acesso 29.06.2019

27. Sarici SU, Gunes O, Koklu E, Serdar MA. Transcutaneous Bilirubin Levels during the First Month of Life in Term and Late-preterm Newborns. J Trop Pediatr. 2017; 63(1):4-9.

28. Skiöld B, Hallberg B, Vollmer B, Ådén U, Blennow M, Horsch S. A Novel Scoring System for Term-Equivalent-Age Cranial Ultrasound in Extremely Preterm Infants. Ultrasound Med Biol. 2019; 45(3):786-794. 